\title{
Hysteresis and reentrant melting of a self-organized system of classical particles confined in a parabolic trap
}

\author{
F. F. Munarin, ${ }^{1,2, *}$ K. Nelissen, ${ }^{1, \dagger}$ W. P. Ferreira, ${ }^{2}$ G. A. Farias, ${ }^{2}$ and F. M. Peeters ${ }^{1, \ddagger}$ \\ ${ }^{1}$ Department of Physics, University of Antwerp, Groenenborgerlaan 171, B-2020 Antwerpen, Belgium \\ ${ }^{2}$ Departamento de Física, Universidade Federal do Ceará, Caixa Postal 6030, Campus do Pici, 60455-760 Fortaleza, Ceará, Brazil
}

(Received 10 July 2007; revised manuscript received 3 December 2007; published 20 March 2008)

\begin{abstract}
The melting of a self-organized system composed of classical particles confined in a two-dimensional parabolic trap and interacting through a potential with a short-range attractive part and a long-range repulsive potential is studied. Different behaviors of the melting temperature are found depending on the strength $(B)$ of the attractive part of the interparticle potential. The melting of a system consisting of small bubbles takes place through a two-step melting process. A reentrant behavior and a thermally induced structural phase transition are observed in a small region of the $(B, \kappa)$ space. A hysteresis effect in the configuration of the particles is observed as a function of temperature. This is a consequence of the presence of a potential barrier between different configurations of the system.
\end{abstract}

DOI: 10.1103/PhysRevE.77.031608

PACS number(s): 64.60.Q-, 64.60.Cn, 61.46.-w, 75.60.Nt

\section{INTRODUCTION}

The study of the properties of self-organized systems has been an important topic of research in recent years. This interest originates from the possibility to control the formation of patterns having an important impact on applications that use large-scale self-assembly to create specific pattern morphologies. These kinds of structures occur in systems from diverse areas including chemistry [1] and biology [2]. In physics, it was predicted that systems which fall into this morphological category are generated by the competition between short-range attraction and long-range repulsion [3]. This competitive interaction appears in many systems, such as magnetic materials [4,5], colloids [6,7], and twodimensional electron systems [8,9]. Experimentally, colloidal systems are one of the most studied systems, which, in combination with theoretical predictions, may lead to the design of novel soft materials and to an understanding of the glass and gel state of matter [10]. Recently, it was observed that a similar type of pattern formation can also arise in particular classes of ultrasoft colloids with a strictly repulsive interparticle potential [11].

Besides presenting a rich variety of cluster types and showing an excellent model for technological applications, colloidal systems have the added advantage of the facility to control the interaction between particles and of real-time imaging of their configuration through video microscopy. A wide variety of studies on colloidal systems were performed in order to understand the structure and dynamics of different kind of systems, such as colloidal particles interacting through a short-range attractive and long-range repulsive potential [12-14]. For instance, a binary system of superparamagnetic colloidal particles that are confined by a twodimensional (2D) water-air interface and exposed to an external magnetic field perpendicular to the interface showed

\footnotetext{
*munarin@fisica.ufc.br

†kwinten.nelissen@ua.ac.be

†francois.peeters@ua.ac.be
}

diverse stable configurations [15]. Moreover, the authors observed that clustering appeared only for one type of particles, instead of both types of particles.

Recently, several models were developed having a small number of interacting particles in order to understand the behavior of colloidal systems as a function of temperature [16-18]. Different melting scenarios were studied extensively in systems consisting of charged particles for a range of different interparticle interaction and trap types. For example, systems of binary charged particles confined by a circular hard-wall potential interacting by a repulsive dipole potential [19], confined by a parabolic trap potential and interacting by a Coulomb interparticle potential [20,21], and nonconfined particles with short-range attraction and longrange repulsion interaction were studied theoretically [22].

Motivated by the increased interest in the behavior of systems of particles that are characterized by a competition between short-range attraction and long-range repulsion, we analyze here the melting of a system composed of a finite number of classical particles interacting through a potential which is composed of a repulsive Coulomb and an attractive exponential term. The particles move in a $2 \mathrm{D}$ plane that are confined by a parabolic trap. The zero-temperature configurations of this system were studied recently by Nelissen et al. [23]. They observed different topological configurations (e.g., ring, stripe, and bubble configurations). From these results arises the question about the stability of those different configurations against thermal fluctuations. This motivated us to study the melting of these ordered configurations and to analyze the effect of the interplay between the short-range and long range interactions on the melting process. We found that if one increases the temperature, some of the bubble configurations exhibit a thermally induced structural phase transition and a remarkable reentrant behavior. In addition, we found that this system exhibits hysteresis behavior for the mean radial displacement when we increase and decrease the temperature and the configuration goes through a thermally induced structural phase transition.

This paper is organized as follows. In Sec. II, we describe the mathematical model and the numerical approach to ob- 
tain the ground-state configurations. In Sec. III we analyze the melting process for different interparticle potentials. Temperature-induced reentrant and hysteresis effects are discussed in Sec. IV. Our conclusions are presented in Sec. V.

\section{NUMERICAL APPROACH}

We consider a 2D cluster with $N$ classical particles interacting through a potential composed of a repulsive Coulomb and an attractive exponential term as in Refs. [22,23]. The particles are kept together by a parabolic potential centered at the origin. The general dimensionless Hamiltonian of the system is written as

$$
H=\sum_{i=1}^{N} r_{i}^{2}+\sum_{i>j=1}^{N}\left(\frac{1}{\left|\vec{r}_{i}-\vec{r}_{j}\right|}-B e^{-\kappa\left|\vec{r}_{i}-\vec{r}_{j}\right|}\right),
$$

where $r_{i} \equiv\left|\mathbf{r}_{i}\right|$ is the distance of the $i$ th particles from the center of the parabolic confinement. Energy and distance are in units of $E_{0}=\left(m \omega_{0}^{2} r_{0}^{2} / 2\right)$ and $r_{0}=\left(2 q^{2} / m \epsilon \omega_{0}^{2}\right)^{1 / 2}$, respectively. Notice that the $B$ parameter determines the exponential contribution in the Hamiltonian and it is responsible for the strength of the attractive part of the interparticle potential [the third term in Eq. (1)]. Temperature is expressed in units of $T_{0}=E_{0} / k_{B}$, where $k_{B}$ is the Boltzmann constant.

The ground-state configuration $(T=0)$ of the twodimensional system was obtained by the Monte Carlo (MC) simulation method (using the standard Metropolis algorithm [24]) extended with the Newton optimization method [25]. The particles were allowed to reach a steady-state configuration after $10^{5}$ simulation steps, starting from different initial random positions. In the same time, we calculate the frequencies of the normal modes of the system using the Householder diagonalization technique [25]. The configuration was taken as final if all frequencies of the normal modes were positive and the energy did not decrease further.

In order to understand the trajectory of each particle correctly, we study the melting of the system using molecular dynamic (MD) simulation. The temperature of system was increased from $T=0$ (ground-state configuration) with successive steps of $\delta T$ and equilibrated at the new temperature during $10^{6} \mathrm{MD}$ steps (with a typical step size of $\Delta t=0.001$ ). After this equilibrium, the average energy was calculated, together with the mean squared radial displacement given by

$$
\left\langle u_{R}^{2}\right\rangle \equiv \frac{1}{N} \sum_{i=1}^{N}\left(\left\langle r_{i}^{2}\right\rangle-\left\langle r_{i}\right\rangle^{2}\right) / \rho^{2},
$$

where $\rho$ is the average interparticle distance at zero temperature. The symbol $\langle\cdots\rangle$ stands for an average over typically $10^{6}-10^{7} \mathrm{MD}$ steps after equilibration of the system.

The system studied exhibits complex configurations [23], such as, e.g., the bubble configuration shown in the inset of Fig. 1, and presents interesting different melting processes. In order to understand better the melting behavior of the system, we analyze the mean radial displacement and angular disorder of the particles of the small bubbles as a function of temperature. In particular, we calculate the radial and angular disorder of each particle with respect to the center of

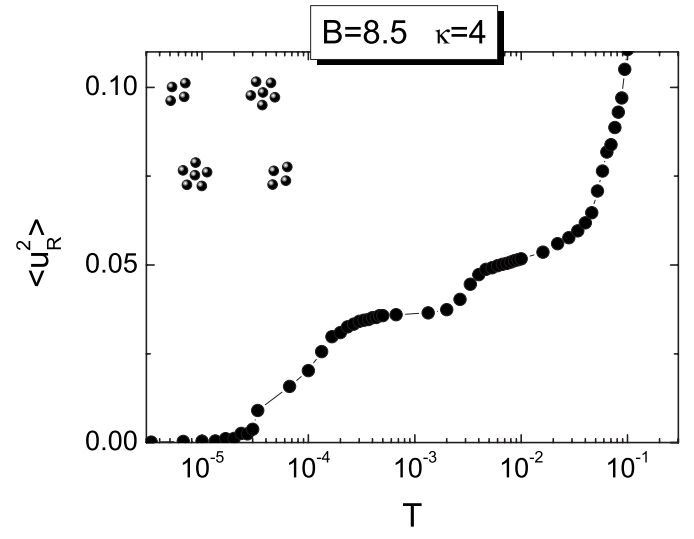

FIG. 1. The mean radial displacement for a system with $N=20$, $B=8.5$, and $\kappa=4$ as a function of temperature given in a logarithmic scale. The ground-state configuration is shown in the inset.

mass of the small bubbles to which it belongs. The mean local radial displacement is defined as

$$
\left\langle u_{R(B)}^{2}\right\rangle \equiv \frac{1}{N_{c}} \frac{1}{N_{s c}} \sum_{j=1}^{N_{c}} \sum_{i=1}^{N_{s c}}\left[\left\langle\left(r_{j}^{\text {c.m. }}-r_{i}\right)^{2}\right\rangle-\left\langle\left(r_{j}^{\text {c.m. }}-r_{i}\right)\right\rangle^{2}\right] / \rho_{B}^{2},
$$

where $N_{c}$ and $N_{s c}$ are the number of small bubbles and the number of particles in each small bubble, respectively. $r_{j}^{c . m .}$ is the distance of the center of mass of the bubble from the center of the confinement potential, and $\rho_{B}$ is the average distance between the particles of the same small bubble at zero temperature.

The angular disorder in the small clusters is studied using the angular intrashell displacement calculated locally. Previously, this property was used to calculate the angular disorder in the whole system $[16,20]$, but here, we will calculate the angular disorder in each small bubble-i.e., with respect to the center of mass of the small agglomerate of particles, which is defined as

$$
\left\langle u_{\theta(B)}^{2}\right\rangle \equiv \frac{1}{N_{c}} \frac{1}{N_{s c}} \sum_{j=1}^{N_{c}} \sum_{i=1}^{N_{s c}}\left[\left\langle\left(\varphi_{i}-\varphi_{i 1}\right)^{2}\right\rangle-\left\langle\left(\varphi_{i}-\varphi_{i 1}\right)\right\rangle^{2}\right] /\left(\varphi_{0}^{s}\right)^{2},
$$

where $i_{1}$ indicates the nearest neighbor in the same bubble and $\varphi_{0}^{s}=2 \pi / N_{s c}$ is the average density of particles in each small bubble at zero temperature.

Due to the rotation of the small bubble with respect to the confinement center, the center of mass of each small bubble is calculated in each MD step. Moreover, we calculate the angular disorder of the center of mass of each small bubble with respect to the center of confinement, which is defined as

$$
\left\langle u_{\theta}^{2}(\mathrm{c} . \mathrm{m} .)\right\rangle \equiv \frac{1}{N_{c}} \sum_{i=1}^{N_{c}}\left[\left\langle\left(\varphi_{i}-\varphi_{i 2}\right)^{2}\right\rangle-\left\langle\left(\varphi_{i}-\varphi_{i 2}\right)\right\rangle^{2}\right] /\left(\varphi_{0}^{c}\right)^{2},
$$

where $i_{2}$ indicates the nearest center of mass and $\varphi_{0}^{c}$ $=2 \pi / N_{c}$ is the average density of small bubbles in the system at zero temperature. 
The melting temperature was determined through a Lindemann-like criterion, which has been widely used for 2D finite-size clusters. This criterion states that melting occurs when $\left\langle u_{R}^{2}\right\rangle$ reaches 0.1 of the interparticle distance at zero temperature [26]. But what is essential is that melting is characterized by a rapid increase of the fluctuation of particles when temperature reaches the melting temperature.

\section{MELTING}

We start analyzing the stability and the behavior of small bubbles as a function of temperature. To do so, we initially consider a cluster with $N=20$ particles, for $B=8.5$ and $\kappa=4$, which is characterized by small bubbles with a different number of particles-i.e., two small bubbles with four particles and two other ones with six particles (see inset in Fig. $1)$. The particles of the four-particle bubbles are arranged in a lozenge-type structure, while the six-particle bubbles present a circular ring-type configuration.

In Fig. 1, the mean radial displacement $\left\langle u_{R}^{2}\right\rangle$ of the particles with respect to the center of confinement is shown as a function of temperature. As can be observed, $\left\langle u_{R}^{2}\right\rangle$ increases gradually with increasing temperature and exhibits plateaus in some temperature intervals. These plateaus are usually an indication of the presence of thermally induced structural transitions before the system melts completely [29]. In order to study more deeply the structural transitions and the general behavior of the system before melting, we will make use of the properties previously defined in Sec. II.

Figure 2 shows the temperature dependence of the mean radial distance of each particle with respect to the center of the confinement potential $\left\langle r_{i}\right\rangle$ [Fig. 2(a)], the mean radial displacement $\left\langle u_{R(B)}^{2}\right\rangle$, and the mean radial distance $\left\langle r_{i(B)}\right\rangle$ of each particle with respect to the center of mass of the small bubbles to which it belongs [Figs. 2(b) and 2(c), respectively]. The temperature dependence of the mean angular intrashell displacement of particles with respect to the center of mass of the small bubbles $\left\langle u_{\theta(B)}^{2}\right\rangle$ and the mean angular intrashell displacement of the center of mass of the small bubbles with respect to the center of the confinement potential $\left\langle u_{\theta}^{2}(\right.$ c.m. $\left.)\right\rangle$ is also shown in Figs. 2(d) and 2(e), respectively. These properties refer to the system with $N=20$ particles, $B=8.5$ and $\kappa=4$. In Figs. 2(a) and 2(c), the open and solid circles represent the positions of the particles in the four- and six-particle bubbles, respectively.

As can be seen in Fig. 2(c) for very small temperatures $\left(T \leq 5 \times 10^{-5}\right)$, particles located at the edge of the six-particle bubbles are not at the same distance from the center of each bubble, which is a consequence of the positional correlation with the other particles in the system. When $T \approx 2 \times 10^{-4}$, Fig. 2(a) indicates that the distance of these particles becomes the same with respect to the center of the confinement potential, while Fig. 2(c) suggests that the ring-type configuration of the six-particle bubbles remains unaffected. Notice that for $T \approx 2 \times 10^{-4}$ (first vertical dotted line-from left to right) particles located at the edge of the six-particle bubbles are rearranged in order that a perfect circular ring-type structure is formed [Fig. 2(c)]. The apparently conflicting results presented in Figs. 2(a) and 2(c) can be understood by the fact that we are considering the average value of the distance of the particles from the center of the confinement potential. The merging of the curves in Fig. 2(a) is a consequence of the rotation of the six-particle bubbles around their respective center, with the angular order within each of them kept unaltered [Fig. 2(d)]. The rotation of the six-particle bubbles becomes possible due to the increased symmetry of the small bubbles with respect to their center.

The same behavior of the six-particle bubbles is also observed in the four-particle bubbles, but at higher temperature. When $T \approx 3.3 \times 10^{-3}$ (second vertical dotted line), the particles of the four-particle bubbles arrange in a perfect circular ring [Fig. 2(c)]. At this temperature, we find that the internal angular order in each four-particle bubble is maintained since $\left\langle u_{\theta(B)}^{2}\right\rangle \approx 0$. Notice that for $T<3.3 \times 10^{-3}$ the lozenge-type structure of the four-particle bubbles remains intact [Fig. 2(c)].

A further increase of temperature brings the system to a drastic structural phase transition in the small bubbles as can be observed in Fig. 2(c) for $T \approx 0.01$ (third vertical dotted line). This figure shows that one particle of the six-particle bubble moves from the center to the edge of the small bubbles, while Fig. 2(d) shows that the angular order of the small bubbles is destroyed around $T \approx 0.01$. However, Fig. 2(a) shows that the position of the small bubbles with respect to the center of the confinement potential remains unaffected. These results indicate that after a thermally induced structural phase transition a local angular melting occurs in the small bubbles. This transition can also be identified as a typical plateau in the $\left\langle u_{R(B)}^{2}\right\rangle$ curve shown in Fig. 2(b) for $T$ $>0.01$. A similar behavior of the $\left\langle u_{R(B)}^{2}\right\rangle$ curve was found earlier [20,29], but in this case, the plateau is a consequence of a structural transition in only one kind of bubble-i.e., six-particle bubbles.

From Fig. 2(b) we find that the small bubbles melt when $T \approx 0.06$ (fourth vertical dotted line), destroying the angular order of the small bubbles with respect to the center of the confinement potential [Fig. 2(e)], and consequently it changes the configuration of the system [Fig. 2(a)]. For $T$ $>0.06$, the value of the mean radial displacement increases sharply and, according to the Lindermann criterion, it reaches the critical value $\left(\left\langle u_{R}^{2}\right\rangle=0.1\right)$ at $T \approx 0.1$, indicating that the system melts (fifth vertical dotted line). It is important to mention that the small bubbles undergo a local rotation before melting indicating that the melting of this system proceeds via a local two-step process. Moreover, we observed that the melting temperature of this system is about 5 times higher than the melting temperature of a pure Coulomb system composed of $N=20$ identical particles $\left(T_{m}=0.012\right)$. This fact indicates that the system composed of small bubbles are more stable than a pure Coulomb system.

In order to observe the influence of the configuration on the melting temperature, we show in Fig. 3 the melting temperature for the total system as a function of the $B$ parameter for a system with $N=20$ and $\kappa=4$. We define in Fig. 3 three different regions associated with ring (I and III) and nonring configurations (II). Configurations of these regions are shown in Fig. 4. From Figs. 3 and 4 we see that for low $B$ values (region I) the system is in a ring configuration, in region II the system is on a stripe or bubble configuration, 


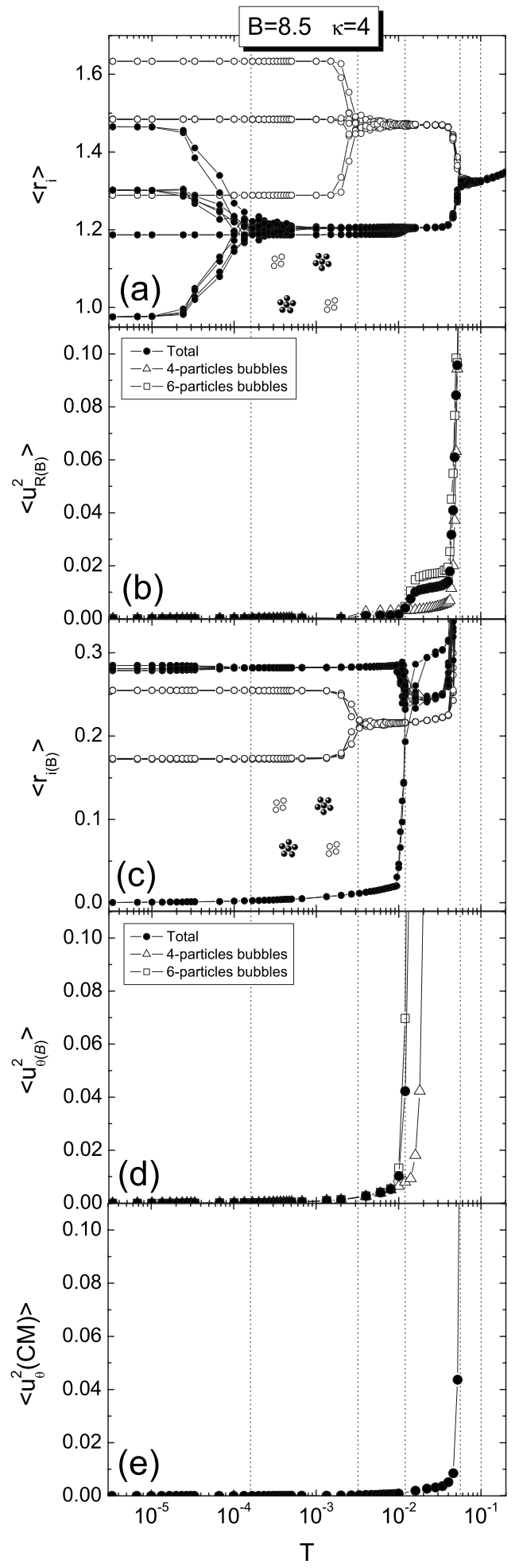

FIG. 2. The temperature dependence of (a) the mean radial displacement of each particle with respect to the confinement center, (b) the mean radial displacement, (c) the mean distance, and (d) the mean angular intrashell displacement of each particle with respect to the center of mass of the small bubbles. (e) The mean angular intrashell displacement of the center of mass of the small bubbles with respect to the confinement center. The open and solid circles in (a) and (c) represent the positions of the particles in the four- and six-particle bubbles, respectively. The temperature is given in a logarithmic scale. and for large $B$ value the system transforms again into a ring configuration (region III). The horizontal dotted line in Fig. 3 indicates the melting temperature of a pure Coulomb system with $N=20$ particles.

Figure 3(a) shows that the melting temperature in the region I decreases with increasing $B$ value, indicating that the system becomes less stable. This behavior is surprising since $B$ is the strength of the short-range attraction [see Eq. (1)], and one may expect that the larger the strength of the attraction, the more packed the particles are, and therefore the higher the melting temperature. This unexpected behavior is a consequence of the fact that the interparticle distance are such that the interparticle potential is purely repulsive in region I. In this region, when the value of $B$ increases, the repulsive potential decreases [see Eq. (1)] and thus also the coupling strength among the particles, which leads to a softening of the normal modes and of the melting temperature.

The melting temperature continues decreasing up to the small value $T_{m} \approx 0.0013$, which is reached for $B=6.0$-i.e., one order of magnitude smaller than the melting temperature of a pure Coulomb system $T_{m}=0.012$. For $B>6.0$, the attraction between the particles starts to influence the system behavior and consequently the melting temperature increases due to the increase of the packing of the particles. For $B$ $=6.8$, the attraction dominates the interaction potential between particles and the system changes its configuration from rings to stripes or bubbles (vertical dotted line between regions I and II).

Region II is composed of several kinds of configurations; some of them are shown in Figs. 4(b)-4(f). Notice that the number of small bubbles in region II decreases with increasing $B$. This is a consequence of the fact that the effective repulsion potential between particles decreases as a function of $B$ [see Eq. (1)]. Thus, more particles can occupy a small bubble, decreasing the number of small bubbles and therefore increasing the distance between them as a function of $B$. As a consequence, the required energy for a particle to overcome the potential barrier between small bubbles increases as a function of $B$ as well as the melting temperature as is apparent in region II of Fig. 3(a). The melting temperature in region II continues increasing as a function of $B$ up to $B$ $=14$ (vertical dotted line between regions II and III). At this $B$ value, the system melts at $T_{m}=1.18$-i.e., two orders of magnitude higher than the melting temperature of a pure Coulomb system-confirming that the system composed of bubbles is more stable than a Coulomb system. For $B=14$, the system transits back to a ring configuration, indicating that the strength of the short attraction potential between the particles is large enough to agglomerate all particles in a short distance. Since there are no small clusters present and consequently no large potential barrier for particles to overcome, the melting temperature decreases drastically and at the same time the system changes its configuration as shown in Fig. 3(a).

In region III an interesting behavior of the melting temperature curve can be observed. Figures 3(a) and 3(b) show that the melting temperature increases gradually as a function of $B$ up to $B=60$ when it begins to decrease asymptotically. It is important to observe that this melting temperature behavior occurs when the system has the ring structure; i.e., 

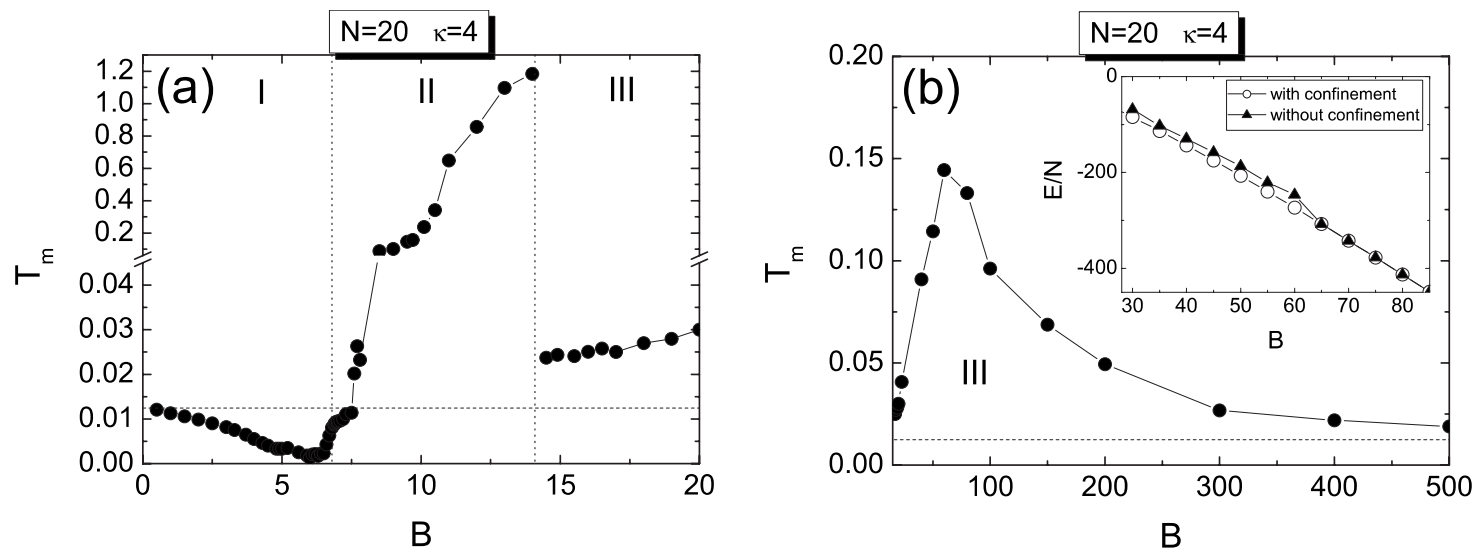

FIG. 3. The melting temperature $\left(T_{m}\right)$ of the total system (i.e., transition to the disordered phase) as a function of $B$ for a system with $N=20$ particles and $\kappa=4$. (a) is for small and (b) is for large $B$ values. The horizontal dotted line indicates the melting temperature of a pure Coulomb system with $N=20$ particles. The energy per particles of this system with and without confinement potential are shown as the inset of (b).

the melting temperature of the system shows a large variation for the same structure. As in region II, the melting temperature increases in region III as a consequence of the decrease of the repulsion between particles and thus the system decreases its size due to the influence of the confinement potential. We observe a difference in the size of the system from Figs. 4(g) and 4(h), which show two configurations of region III in the same scale. For $B=60$, the system is so small that the confinement potential is no longer relevant (i.e., it is too weak) to compress the system. The system becomes internally stabilized solely due to the interparticle potential. The potential barrier preventing the hopping of particles between the rings decreases with increasing $B$, destabilizing the system and therefore decreasing the melting temperature. In order to confirm that the confinement potential does not influence the system for $B>60$, we plot as an inset to Fig. 3(b) the minimum energy per particle for the system described by Eq. (1) with and without confinement potential for $N=20$ and $\kappa=4$. The energy of these two systems with and without confinement potential becomes the same around $B=60$, confirming that the system of bubbles is no longer influenced by the confinement potential for $B$ $>60$.

We checked that the general behavior of the melting temperature of the total system $\left(T_{m}\right)$ shown in Figs. 3(a) and 3(b) for the cluster with $N=20$ particles was also observed for $N=10,30,40$, and 50 particles, but now for a different value of the $B$ parameter.

\section{STRUCTURAL BEHAVIOR}

Recently, colloidal systems exhibited several new and interesting features, such as reentrant behavior $[6,18,19,27]$ and a hysteresis effect [28]. In this section, we show for a specific short-range interaction that our system exhibits a different reentrant effect and a hysteresis behavior as a function of temperature.

\section{A. Reentrant behavior}

The temperature dependence of the mean-squared radial displacement $\left\langle u_{R}^{2}\right\rangle$ is shown in Fig. 5(a) for a system with

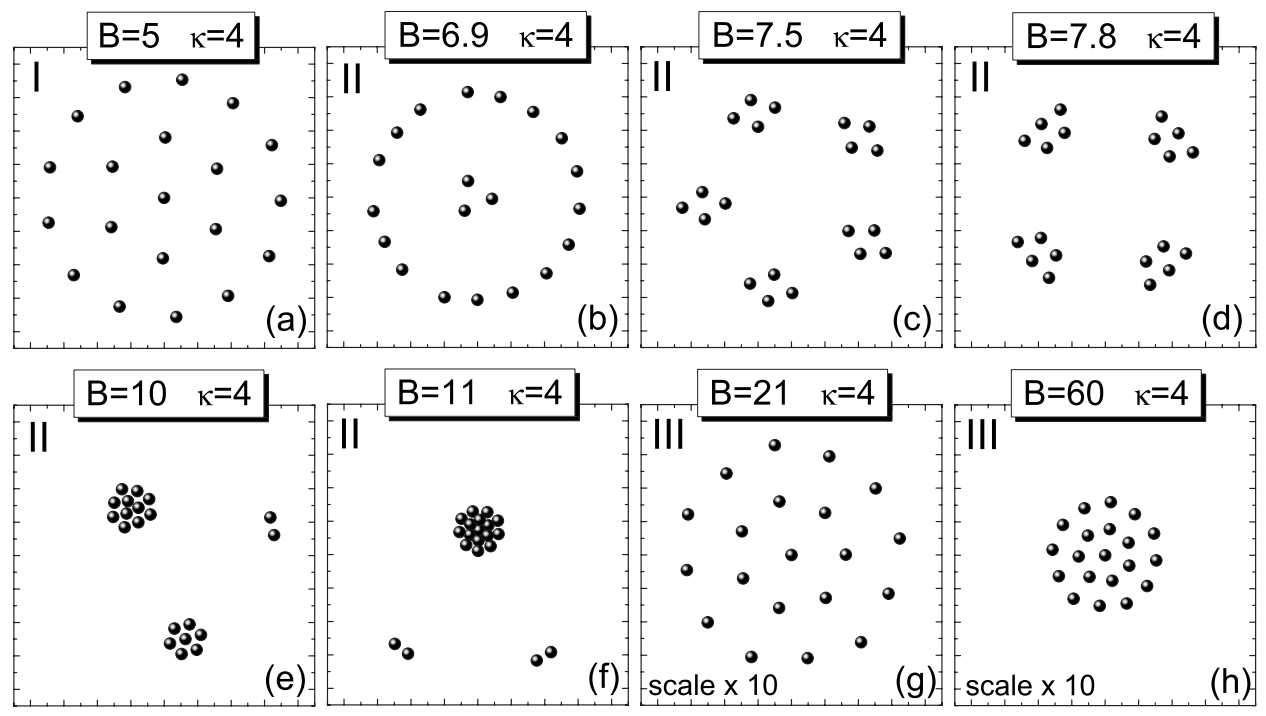

FIG. 4. Snapshots of the ground-state configuration for a system with $N=20$ particles, $\kappa=4$ and different values of $B$. 


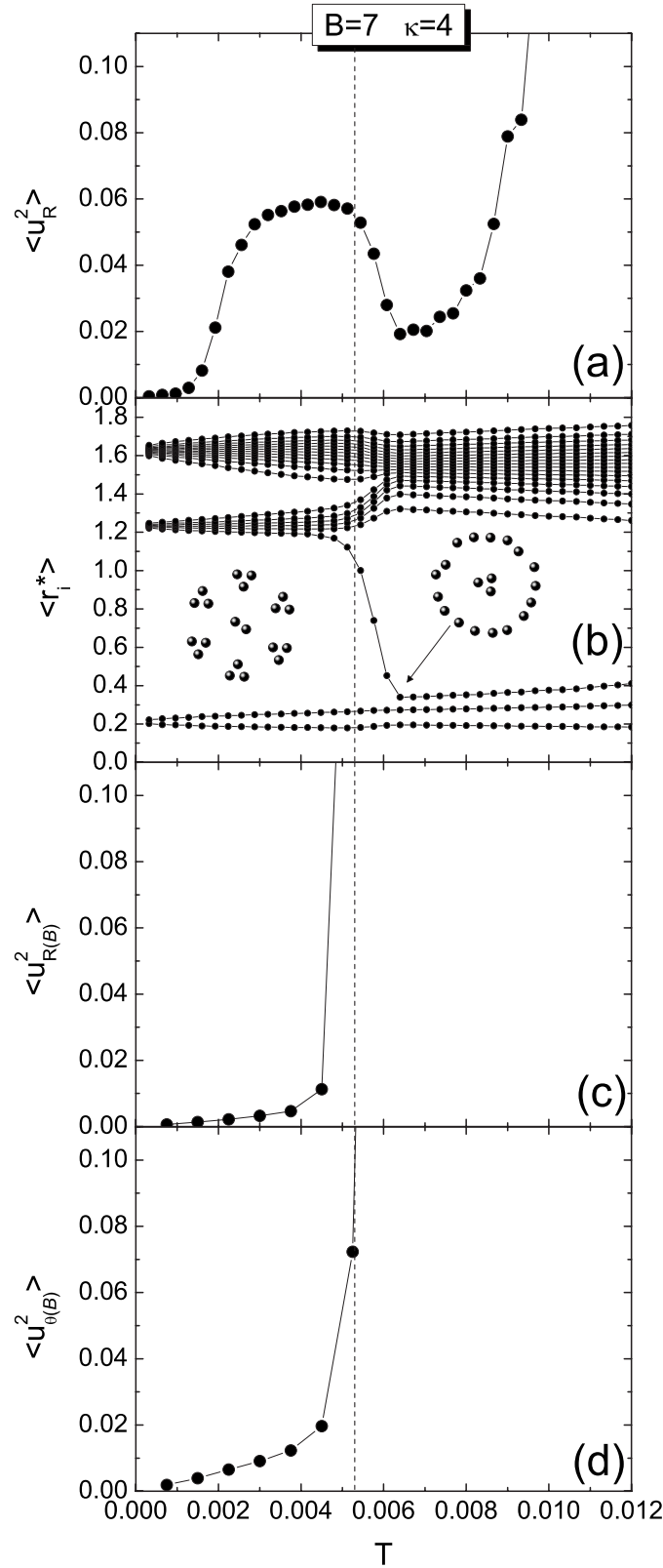

FIG. 5. (a) The mean radial displacement $\left(\left\langle u_{R}^{2}\right\rangle\right)$, (b) the mean distance of each particle from the center of the confinement potential, (c) the mean radial displacement, and (d) the angular intrashell displacement with respect to the center of mass of the small bubble for a system with $N=20, B=7$, and $\kappa=4$.

$B=7$ and $\kappa=4$. When the temperature increases from $T=0$ to $T=0.003$, the value of $\left\langle u_{R}^{2}\right\rangle$ increases considerably until it reaches a semiplateau and remains almost constant until $T$ $=0.0053$ (dashed line). For $T>0.0053$, we observe that the value of $\left\langle u_{R}^{2}\right\rangle$ decreases rapidly before it increases sharply, indicating that the system melts. This reentrant behavior was observed in both experimental $[6,27]$ and theoretical $[18,19]$ studies, and in the present case, it is due to an increase of the stability of the whole system caused by an increase of the symmetry of the system configuration. In other words, the value of the mean radial displacement decreases after $T$ $=0.0053$ as a result of the change in the configuration of the system.
In order to confirm that the reentrant behavior is caused by a change in the configuration, we plot in Fig. 5(b) the position of the particles with respect to the center of the confinement potential as a function of temperature. Specifically, in each MD step we organize the distance of each particle in such a way that $r_{1}, r_{2}, r_{3}, \ldots, r_{N}$ correspond to the first, second, third,..., $N$ th closest particles from the center of the cluster, respectively. After that, we calculate the average of each closest distance $\left\langle r_{i}^{*}\right\rangle$ for each temperature and we present it as a function of temperature in Fig. 5(b). As can be seen, the system remains in the bubble configuration from $T=0$ to $T=0.0053$. We will label this configuration as $\left(2 ; 6(3)^{B}\right)$, which means that there is 1 ring of 2 particles and 6 bubbles of 3 particles [see the left inset of Fig. 5(b)]. For $T>0.0053$, a particle leaves the edge and moves to the center of the system. The configuration changes from the bubble $\left(2 ; 6(3)^{B}\right)$ to the ring configuration $(3 ; 17)$, which means that there is a ring of 3 particles and another with 17 particles. The outer ring has a nonzero width. We observe from Figs. $5(\mathrm{a})$ and 5(b) that the value of the mean radial displacement decreases when the configuration changes from $\left(2 ; 6(3)^{B}\right)$ to $(3 ; 17)$ and increases rapidly when the temperature approaches the melting temperature of the ring configuration. Therefore, this change of configuration is a structural transition which stabilizes the system. This transition occurs before the system is completely melted and is a thermally induced structural phase transition. This interesting phenomenon was found in diverse previous studies [29-31], but in this case, it is the result of the increase of disorder in small bubbles when the temperature approaches $T=0.0053$.

In order to better understand the local disorder of the particles, we present in Figs. 5(c) and 5(d) the mean radial displacement and angular disorder of the particles of the small bubbles as a function of temperature, respectively, as defined in Sec. II. As we can see, the mean radial displacement and the angular disorder of the particles increase simultaneously very dramatically in each bubble when the temperature approaches $T=0.0053$ (dotted vertical line). The thermal fluctuation permits a particle to overcome the potential barrier allowing it to move to the center and consequently changing the system configuration from $\left(2 ; 6(3)^{B}\right)$ to $(3 ; 17)$.

Reentrant behavior was found in a small region of $(\kappa, B)$ space which is shown in Fig. 6. We found that this interesting feature is the result of the interparticle potential profile which is illustrated in the inset of Fig. 6 for some values of $B$ and $\kappa$. We observe that the interparticle potential for the system with $B=7$ and $\kappa=4$ is not so repulsive as that for $B=6$ and $\kappa=5$ and it is not so attractive as for $B=8$ and $\kappa=3$. Therefore, systems which have values of $B$ and $\kappa$ close to 7 and 4 , respectively, are ideal to exhibit a reentrant behavior due to the characteristic of agglomerating particles that are sufficiently weakly bound to allow particles to overcome the potential barrier when temperature increases-i.e., a thermally induced structural phase transition.

The reentrant phenomenon present for the cluster with $N=20$ particles was also observed for other values of $N$-e.g., for $N=30$ particles. The general behavior of $\left\langle u_{R}^{2}\right\rangle$ shown in Fig. 5(a) was also found for $N=30$ particles, but now for a slightly different region in $(\kappa, B)$ space, as well as the thermally induced structural phase transition. 


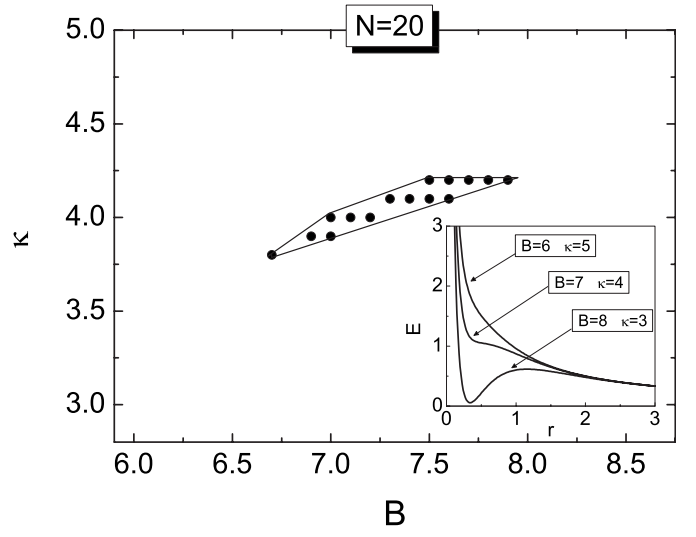

FIG. 6. Phase diagram in $(B, \kappa)$ parameter space showing the reentrant behavior for $N=20$ particles (solid symbols are the calculated values). The profile of the interparticle potential for some relevant $(B, \kappa)$ values is shown in the inset.

\section{B. Hysteresis behavior}

Figure 7 shows $\left\langle u_{R}^{2}\right\rangle$ when we decrease temperature after the system changed its configuration to $(3 ; 17)$ for $N=20, B$ $=7$, and $\kappa=4$. The mean radial displacement is calculated and the temperature is increased from $T=0$ to $T=0.008$ (solid circles). In $T=0.008$, the system does not melt, but reaches a different configuration from the $T=0$ configuration. After that, we decreased temperature until $T=0$ (red square symbols) and increased it again up to $T=0.008$ (green triangle symbols). Notice that at $T=0.008$ the system reaches a different configuration from the ground-state one and that the $\left\langle u_{R}^{2}\right\rangle$ behavior is very different when temperature is decreased indicating that the system gets stuck in a metastable state. This phenomenon is very interesting because the system exhibits a hysteresis effect in the mean radial displacement as a function of temperature when we decrease the temperature after a thermally induced phase transition-i.e., after a change of configuration. This interesting behavior is a consequence of the fact that the $(3 ; 17)$ configuration is stable, although it has a larger potential energy than that of the $\left(2 ; 6(3)^{B}\right)$ configuration, and is separated from the $\left(2 ; 6(3)^{B}\right)$ configuration by a high-energy saddle point.

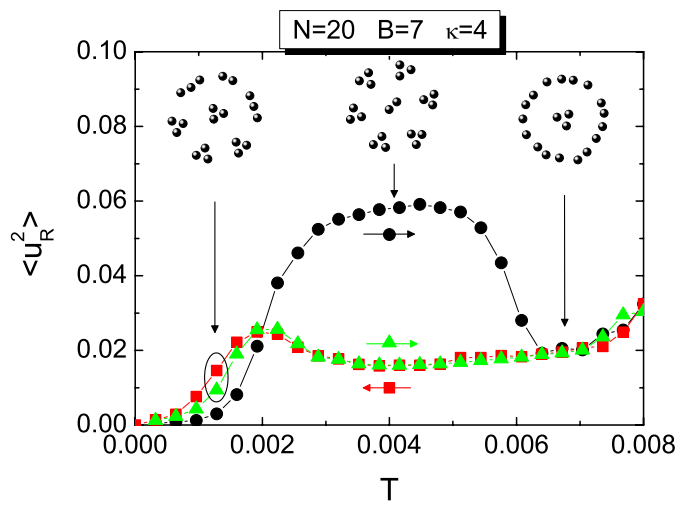

FIG. 7. (Color online) The mean radial displacement for a cluster with $N=20, B=7$, and $\kappa=4$ for increasing (black ball and green triangle symbols) and decreasing temperature (red squared symbols).

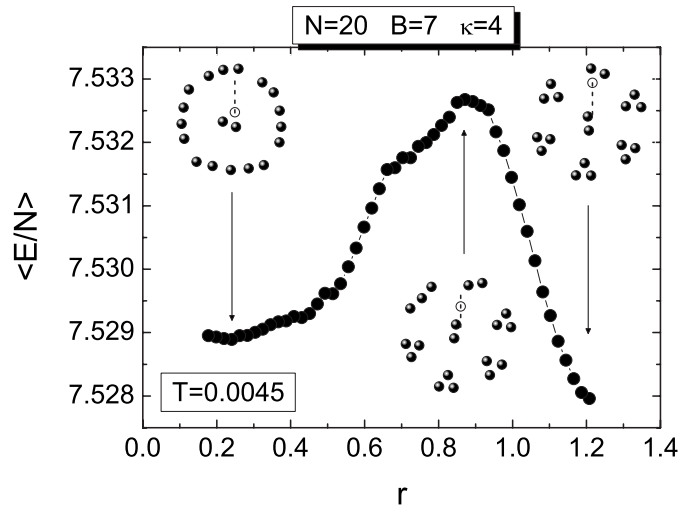

FIG. 8. The mean energy per particle as a function of the distance of the marked particle (particle in the inset indicated by a open circle) from the center of the confinement potential for the cluster with $B=7, \kappa=4$, and $N=20$ particles and fixed temperature $T=0.0045$. The configurations at the three local energy are shown in the inset as well as the trajectory of the marked particle (dashed line).

In order to visualize this saddle point in the potential energy landscape between the $(3 ; 17)$ and $\left(2 ; 6(3)^{B}\right)$ configuration, we fixed one particle at the edge of the $\left(2 ; 6(3)^{B}\right)$ configuration at $T=0.0045$ and moved it in the direction of the center and at the same time allowing all the other particles to relax to their equilibrium positions. We calculated the average energy per particle of the system as a function of the position of the fixed particle (Fig. 8). In this simulation we used the Monte Carlo technique in order to obtain the ground-state configuration and to make an average of the particle energy of several MC steps (around $10^{7}$ ). This average energy per particle is shown in Fig. 8 as a function of the distance of the marked particle (open symbol in the inset and this particle is moved along the thin dashed line) with respect to the center of the confinement potential for a fixed temperature $T=0.0045$.

In Fig. 8, we observe that the average energy per particle increases sharply when the marked particle is moved from $r=1.2$ to $r=0.9$. After that, the mean energy of the system decreases continuously until $r \approx 0.3$ and remains almost constant until $r=0.17$. The $(3 ; 17)$ configuration is found when $r=0.21$. Notice that the $(3 ; 17)$ configuration has the second lowest energy of the whole simulation and is stable. Thus, when we increase the temperature of the $\left(2 ; 6(3)^{B}\right)$ configuration such that there is enough thermal energy for a particle to overcome the potential energy saddle point, of $T=0.007$, the system changes its configuration to $(3 ; 17)$ and is locked into this lowest-energy configuration. Consequently, the system remains in this metastable configuration even when temperature is decreased down to $T=0$.

\section{CONCLUSION}

In this paper we investigated the dependence of the melting temperature of a system composed of classical particles, interacting through an interparticle potential with a shortrange attractive part and a long-range repulsive part, confined by a parabolic trap. The system of bubbles showed 
diverse and nontrivial behaviors as a function of temperature. We observe that the small bubbles rotate and melt locally before the melting of the total system, indicating that the melting phenomenon of this system proceeds in a two-step process-i.e., a two-step process in the behavior of the small bubbles. Moreover, the system exhibits a thermally induced structural phase transition in the small bubbles before it melts completely.

The melting temperature curve showed diverse behaviors as a function of the $B$-parameter. In general, the melting temperature decreases as a function of $B$ for pure repulsive systems and increases for a packed system-e.g., stripes and bubbles. The highly packed system shows a different behavior of the melting temperature as a function of $B$. For large $B$ values we found that the confinement potential is no longer important in the melting process.

The mean radial displacement $\left\langle u_{R}^{2}\right\rangle$ showed a reentrant behavior as a function of temperature. We found that this behavior is a consequence of a thermally induced structural phase transition which stabilizes the system before it melts. This structural transition occurs due to the rapid increase of the local disorder in the small bubbles of the system. This reentrant behavior is found for a restricted set of values for the $B$ and $\kappa$ parameters which define the attractive part of the potential. These values that lead to a reentrant behavior are shown in a phase diagram.

A hysteresis effect was observed in the structural and dynamical behavior of the system as a function of temperature. It was shown that this behavior is a consequence of the existence of a high-energy saddle point in the potential energy landscape between the two lowest-energy configurations.

\section{ACKNOWLEDGMENTS}

F.F.M., W.P.F., and G.A.F. were supported by the Brazilian National Research Councils CNPq and CAPES and the Ministry of Planning (FINEP). Part of this work was supported by the Flemish Science Foundation (FWO-Vl).
[1] D. Philp and J. F. Stoddart, Angew. Chem. Int. Ed. Engl. 35, 1154 (1996).

[2] H. C. Chang, T. L. Lin, and G. G. Chang, Biophys. J. 78, 2070 (2000).

[3] R. P. Sear and W. M. Gelbart, J. Chem. Phys. 110, 4582 (1999).

[4] B. P. Stojković, Z. G. Yu, A. L. Chernyshev, A. R. Bishop, A. H. Castro Neto, and N. Grønbech-Jensen, Phys. Rev. B 62, 4353 (2000).

[5] B. P. Stojković, Z. G. Yu, A. R. Bishop, A. H. Castro Neto, and N. Grønbech-Jensen, Phys. Rev. Lett. 82, 4679 (1999).

[6] R. Bubeck, C. Bechinger, S. Neser, and P. Leiderer, Phys. Rev. Lett. 82, 3364 (1999).

[7] Q. H. Wei, C. Bechinger, D. Rudhardt, and P. Leiderer, Phys. Rev. Lett. 81, 2606 (1998).

[8] J. Schmalian and P. G. Wolynes, Phys. Rev. Lett. 85, 836 (2000).

[9] E. Fradkin and S. A. Kivelson, Phys. Rev. B 59, 8065 (1999).

[10] F. Sciortino, Nat. Mater. 1, 145 (2002).

[11] B. M. Mladek, D. Gottwald, G. Kahl, M. Neumann, and C. N. Likos, Phys. Rev. Lett. 96, 045701 (2006).

[12] A. I. Campbell, V. J. Anderson, J. S. van Duijneveldt, and Paul Bartlett, Phys. Rev. Lett. 94, 208301 (2005).

[13] P. J. Lu, J. C. Conrad, H. M. Wyss, A. B. Schofield, and D. A. Weitz, Phys. Rev. Lett. 96, 028306 (2006).

[14] E. V. Shevchenko, D. V. Talapin, N. A. Kotov, S. O'Brien, and C. B. Murray, Nature (London) 439, 55 (2006).

[15] N. Hoffmann, F. Ebert, C. N. Likos, H. Lowen, and G. Maret, Phys. Rev. Lett. 97, 078301 (2006).

[16] V. M. Bedanov and F. M. Peeters, Phys. Rev. B 49, 2667 (1994)
[17] M. Kong, B. Partoens, and F. M. Peeters, New J. Phys. 5, 23 (2003).

[18] I. V. Schweigert, V. A. Schweigert, and F. M. Peeters, Phys. Rev. Lett. 84, 4381 (2000).

[19] K. Nelissen, B. Partoens, I. Schweigert, and F. M. Peeters, Europhys. Lett. 74, 1046 (2006).

[20] W. P. Ferreira, F. F. Munarin, G. A. Farias, and F. M. Peeters, J. Phys.: Condens. Matter 18, 9385 (2006).

[21] W. P. Ferreira, F. F. Munarin, K. Nelissen, R. N. Costa Filho, F. M. Peeters, and G. A. Farias, Phys. Rev. E 72, 021406 (2005).

[22] C. J. Olson Reichhardt, C. Reichhardt, I. Martin, and A. R. Bishop, Physica D 193, 303 (2004).

[23] K. Nelissen, B. Partoens, and F. M. Peeters, Phys. Rev. E 71, 066204 (2005).

[24] N. Metropolis, A. W. Rosenbluth, M. N. Rosenbluth, A. M. Teller, and E. Teller, J. Chem. Phys. 21, 1087 (1953).

[25] V. A. Schweigert and F. M. Peeters, Phys. Rev. B 51, 7700 (1995).

[26] Yu. E. Lozovik and V. M. Fartzdinov, Solid State Commun. 54, 725 (1985); V. M. Bedanov, G. V. Gadiyak, and Yu. E. Lozovik, Phys. Lett. 109A, 289 (1985).

[27] C. Bechinger, Q. H. Wei, and P. Leiderer, J. Phys.: Condens. Matter 12, A425 (2000).

[28] C. J. Olson Reichhardt, C. Reichhardt, and A. R. Bishop, Europhys. Lett. 72(3), 444 (2005).

[29] W. P. Ferreira, B. Partoens, F. M. Peeters, and G. A. Farias, Phys. Rev. E 71, 021501 (2005).

[30] D. Tomecka, B. Partoens, and F. M. Peeters, Phys. Rev. E 71, 062401 (2005).

[31] G. Coupier, C. Guthmann, Y. Noat, and M. Saint Jean, Phys. Rev. E 71, 046105 (2005). 